Instituto Internacional de Investigación y Desarrollo Tecnológico Educativo INDTEC, C.A.

DOI: https://doi.org/10.29394/Scientific.issn.2542-2987.2019.4.E.9.153-172

OAI-PMH: http://www.indteca.com/ojs/index.php/Revista Scientific/oai

Artículo Original / Original Article

\title{
Gestión administrativa y de calidad en los centros escolares de los Chorrillos
}

Autora: Rocío Soledad Riffo San Martín Universidad Miguel de Cervantes, UMC rocio.riffo@profe.umcervantes.cl Talca, Chile http://orcid.org/0000-0002-3519-500X

\section{Resumen}

El trabajo referido a la gestión administrativa y de calidad en los centros escolares ubicados en Chorrillos, correspondientes a la jurisdicción de la Unidad de gestión Educativa Local (UGEL) 07, pretende indagar sobre la influencia de la gestión administrativa sobre los procesos de calidad. La investigación se desarrolló bajo un paradigma cuantitativo, tipo de investigación básica sustantiva, con un diseño que implica un diseño transversal de nivel explicativo, el cual consiste en aplicar un instrumento, a dos escalas de opinión, a ochenta (80) docentes. De los resultados obtenidos se obtiene que la mayoría de las profesoras de educación inicial han desarrollado competencias administrativas lo que corresponde a cerca de un $70 \%$ de la muestra y el $85 \%$ de las profesoras indican que la gestión es de buena calidad, por lo tanto, de la investigación se concluye que en las actividades desarrolladas se observa buena gestión y de calidad dentro de los centros escolares seleccionados.

Palabras clave: competencias del docente; educación; calidad de la educación.

Cómo citar este artículo:

Riffo, R. (2019). Gestión administrativa y de calidad en los centros escolares de los Chorrillos. Revista Scientific, 4(Ed. Esp.), 153-172, e-ISSN: 2542-2987. Recuperado de: https://doi.org/10.29394/Scientific.issn.2542-2987.2019.4.E.9.153-172

Fecha de Recepción:

10-05-2019
Fecha de Aceptación:

17-07-2019
Fecha de Publicación:

05-09-2019 


\title{
Administrative and quality management in schools in the Chorrillos
}

\begin{abstract}
The work referred to administrative and quality management in schools located in Chorrillos, corresponding to the jurisdiction of the Local Educational Management Unit (UGEL) 07, seeks to investigate the influence of administrative management on quality processes. The research was carried out under a quantitative paradigm, a type of substantive basic research, with a design that implies an explanatory cross-sectional design, which consists of applying an instrument, at two scales of opinion, to eighty (80) teachers. From the results obtained, it is obtained that most of the initial education teachers have developed administrative competencies, which corresponds to about $70 \%$ of the sample and $85 \%$ of the teachers indicate that the management is of good quality, therefore, from the investigation it is concluded that in the activities developed good management and quality is observed within the selected schools.
\end{abstract}

Keywords: teacher qualifications; education; educational quality.

Date Received:

10-05-2019
Date Acceptance:

$17-07-2019$
Date Publication:

05-09-2019 


\section{Introducción}

La administración es un proceso que se norme y se regula de acuerdo a las circunstancias, que aunque existan estamentos legales predeterminados para el quehacer educativo, no siempre se podrá dar el mismo tratamiento a todas las situaciones que imperen, esto dependerá de lo interno y externo que fluctué en cada institución educativa.

Así, el director como gerente del quehacer educativo de educación básica debe conformar un ambiente laboral que les permita a las personas que allí laboran alcanzar la eficiencia de las metas planteadas, es de hacer notar que dentro de la administración.

De igual forma, López (2019), señala que:

[...] El docente es una persona que cultiva sensibilidad humana, paciencia y tolerancia con un alto grado de aceptación por la diversidad de sus estudiantes, partiendo de la observación y previa evaluación de sus alumnos, con la finalidad de afianzar estrategias diversas que permitan la concreción y consolidación de la meta educativa para la formación integral con niveles aceptables de calidad en la educación primaria (pág. 286).

Por otra parte, Chiavenato (2010): aborda los avances de la administración, como "un cuerpo teórico que permite el cumplimiento cabal de los objetivos institucionales donde está inmerso el capital humano y la estructura organizativa de la empresa" (pág. 334); es decir, la dedicación del ser humano por hacer las cosas bien permitirá que estos logros se alcance un el trabajo coordinado, consensuado y sobre todo lograr los objetivos institucionales.

Tomando en cuenta lo antes planteado, se puede decir que toda organización tiene como punto esencial, esto es, su capital humano de allí se puede planear, organizar, ejecutar y controlar, dando resultados cónsonos con las metas propuestas por la organización. 


\section{Desarrollo}

\subsection{La competencia Administrativa del Director y sus Fundamentos}

Teóricos

El proceso educativo busca desarrollar las habilidades y destrezas de los educandos a fin de resolver las múltiples ambigüedades en la vida diaria, por ello el trabajo colaborativo de todos los que hacen vida dentro del quehacer educativo es fundamental para producir los cambios de la organización. Esa interacción del personal en función de objetivos preestablecidos es lo que insta el fenómeno administrativo, donde todos direccionan hacia un fin determinado, consustanciados con la dinámica social donde esta imbuido el ser humano.

Por ello, considera Reyes (2015), la administración: "es la técnica dirigida a dar resultados positivos a la empresa y a todos los que cohabitan en la misma" (pág. 85). De acuerdo a lo pautado, los administradores son quienes deben desarrollar la competencia administrativa, es decir, deben codificar cada una de las tareas encaminadas durante el proceso educativo para lograr los objetivos planteados. De allí que Jerez (2016), resalta que: "eficacia en su puesto de trabajo se basa principalmente cuando la persona administra al personal, es decir, cuando un trabajador ingresa a una empresa colabora en actividades sencillas y progresivamente se va incorporando en otras más complejas" (pág. 176).

\subsection{Desde la visión educativa}

Desde la perspectiva educativa, el éxito de un trabajador en una organización en un momento determinado no es prueba fehaciente para garantizar el éxito del mismo en dicho puesto, porque no se toman en cuenta su relación con el ambiente que lo circunda y por ende le repercute.

De acuerdo a esta realidad se inician las pruebas psicométricas que miden diversos aspectos como la inteligencia, socialización, entre otros factores esenciales desde el momento en que se está seleccionando el 
personal a fin de buscar resultados positivos en las funciones que deberá asumir. La gestión por competencia corresponde con un sistema que evalúa las destrezas, necesidades e intereses, entre otros aspectos que son fundamentales para un puesto de trabajo, logrando los objetivos planteados desarrollando la competitividad positiva en la organización.

A esta situación se le adiciona el valor agregado de la estrategia utilizada para administrar los recursos humanos e inducirlos de acuerdo a los objetivos propuestos, es fundamental, conformar un proceso donde todos los compenetrados y la institución ganen, partiendo de las conductas de entrada con que cuenta dicho empleado para llevar a cabo su trabajo eficientemente; en tanto, Bonilla (2016), sostiene que la función de: "la gestión del capital humano debe estar en la búsqueda del personal idóneo que cuente con las características necesarias para llevar a cabo con eficiencia y eficacia la labor planteada" (pág. 172).

En consecuencia, la competencia es un elemento fundamental que le permite al trabajador cumplir sus labores a partir de varias técnicas o estilos de manera efectiva. A esto se adiciona, que las competencias le permiten al trabajador a partir de sus habilidades y destrezas, dominar un puesto de trabajo efectivamente, situación que se evidencia constantemente.

De acuerdo a los planteamientos antes mencionados, puede resaltarse que la competencia administrativa son las habilidades, aptitudes, actitudes y otros elementos fundamentales de cada trabajador en su puesto de trabajo, evidenciando las capacidades humanas, profesionales necesarias para el desarrollo efectivo dentro de una organización que esta encauzada en el logro de objetivos ya planteados.

En este mismo orden de ideas, Beorlegui (2006), hace referencia a que: "algunas de nuestras características son innatas, transmitidas por herencia genética (naturaleza), y otras son adquiridas por aprendizaje, por contagio del entorno (cultura)" (pág. 140). A nivel educativo, implica que los gerentes de las 
instituciones educativas ponen de manifiesto el enfoque por competencia se cuándo se indaga como ha laborado en situaciones similares en el pasado dentro del mismo entorno educativo.

\subsection{Características de la competencia administrativa}

Hablar de la administración implica decodificar las diferentes tareas por parte de cada trabajador, donde debe utilizarse los recursos de manera efectiva a fin de alcanzar las metas preestablecidas. En el quehacer educativo, el director o gerente educativo deben desarrollar tareas administrativas de manera eficiente y eficaz a fin de lograr que todos los actores y autores de la comunidad educativa trabajen por un fin común.

Asimismo, la administración en el campo del talento humano permite de acuerdo Ahumada (1990): "organizar el personal darle el trato que se merece, indicando las funciones que deben desarrollar, estableciendo una comunicación fluida y horizontal a fin de que puedan desarrollar destrezas y habilidades para que alcance resultados óptimos para la organización" (pág. 41).

A esto se adiciona que la administración del capital humano, de acuerdo a lbáñez (2015a), se encarga de:

[...] Administrar las destrezas, vivencias, aspectos relativos a la salud, entre otros, con el fin de que cumplan con los objetivos específicos de la organización de esta manera, los beneficios serán tanto para la organización, el individuo y la sociedad donde éste se encuentra inmerso (pág. 47).

Asimismo, un director debe poseer una serie de competencias o habilidades administrativas como son: Destrezas, manejo en las tareas específicas solicitadas por el ente rector como es el Ministerio de Educación en cuanto a la entrega de documentos que visualicen el desempeño institucional, matrícula. 
Asimismo, debe estar consonó con el manejo del vocabulario propio de la instancia a la que está inmerso, en cuanto a la gestión pedagógica, administrativa, financiera, entre otros aspectos que soliciten las autoridades de la Unidad de gestión Educativa Local (UGEL) y el Ministerio de Educación. Tener presente la manera como se manifiesta ante una situación en particular, por ejemplo, como se socializa con padres y representantes, con miembros de la comunidad, las alianzas estratégicas, entre otras.

La disposición de hacer las tareas de manera eficiente y eficaz, como el ejemplo institucional debe tener racionalidad y emocionalmente estable en cada situación que se presente. Debe tener preciso las metas que dese alcanzar a fin de motivar al resto del personal para conseguir las metas, orientando ese logro, sin dejar a un lado el aspecto afectivo esencial en todas las relaciones interpersonales.

Exaltar los valores ante el resto del personal, la comunidad, padres y representantes, eventos que deben estar centrados en la ética y la moral, es decir, en todo momento debe estar presente la honestidad, responsabilidad, entre otros, enfatizando que es el ejemplo, el ente rector institucional que todo deben seguir, el director es el ejemplo a seguir. Actualmente, a nivel del Perú se adicionan una serie de características que deben estar presentes en el personal directivo como:

El personal dará lo mejor de sí para el cumplimiento de sus tareas, el contrato que se firma no la condición necesaria para que el trabajador se esfuerzo en hacer las actividades de manera fluida, todo dependerá de la motivación que exista entre las partes.

El directivo a través de su trabajo pondrá de manifiesto sus vivencias, intereses, destrezas, entre otras, prestando un servicio que será remunerado económicamente para sufragar sus gastos diarios, además esto inducirá a la estabilidad emocional por cubrir sus expectativas.

El talento humano según Ibáñez (2015b): con los conocimientos, 
habilidades y destrezas para cumplir las tareas son pocas, no todos pueden ser gerentes, deben tener la ética y moral para poder ser ejemplo del resto del personal (pág. 47). El entrenamiento y capacitación del personal directivo permitirá que las competencias de conocimiento y habilidad se pongan de manifiesto en cada tarea desarrollado logrando metas, mientras que las competencias de gestión están implícitas en la persona, en su interior, de allí la necesidad la comunicación, motivación, entre otros aspectos que hagan que las mismas fluyan mejorando las estrategias de trabajo.

\subsection{Dimensiones de la Competencia Administrativa}

\subsubsection{Dimensión Planificación}

Dentro de la planificación en las instancias educativas está en asegurar el personal directivo idóneo con las destrezas, vivencias, aptitudes necesarias para cumplir con las tareas asignadas en el entorno educativo. De allí que el directivo debe anticiparse a las metas a lograr en tiempo determinado, por ello debe planificar de manera cónsona sujeto a los objetivos planteados y los procedimientos para alcanzar los mismos, de allí la importancia de partir de un plan general para el plantel.

De acuerdo a Werther (2016), considera que la planificación: "como una serie de acciones para cumplir metas tomando en cuenta el capital humano calificado, la eficiencia y efectividad para cumplir con las tareas" (pág. 32). Es de resaltar que, en el quehacer educativo, es fundamental planificar el quehacer educativo y los recursos humanos que se cuentan para lograr el trabajo académico, por ello, debe planificarse de acuerdo a cada tarea, todos cónsonos con los objetivos institucionales. A esto se adiciona lo planteado por Sovero (2014), quien sostiene que planificar: "consiste en tener visión prospectiva sobre los recursos, personal y la meta a lograr dentro de la organización planteada" (pág. 87).

En el entorno educativo, planificar consiste en avizorar lo que se va a 
realizar en función de acciones que deben regir en cuanto a lo académico, administrativo, institucional, comunitario, financiero, a fin de lograr los objetivos institucionales a través de los procedimientos planteados para cada acción.

A esto se adiciona lo planteado por la Organización de las Naciones Unidas para la Educación, la Ciencia y la Cultura (UNESCO), en el año 2009 en cuanto a la planeación educativa a través de los siguientes eslabones: Se diagnóstica, para identificar debilidades que afectan a la institución a fin de determinar las posibles respuestas a las problemáticas presentadas.

En este contexto y de acuerdo a lo expuesto, se establecen las acciones a seguir, sus metas educativas que deben partir del plan, del programa y de los proyectos estableciendo progresivamente las funciones y los tiempos en que deben cumplirse cada actividad. Por otro lado, el acto técnico administrativo, que induce a dar el ejecútese por parte de la autoridad educativa quien es la que autoriza para aprobarla, sin dejar de lado lo referente a la puesta en marcha de cada acción para el logro de los objetivos planteados.

\subsubsection{Dimensión Organización}

De acuerdo a Muñoz (2014a), la organización implica: "la adecuación de las actividades que le permiten a la institución educativa alcanzar las metas planteadas, a partir de una estructura organizativa consolidada, mediación, entre otras fortalezas que conlleven a crear un clima organizacional favorable" (pág. 67).

Todo directivo de una institución educativa debe tener incluido en su proceso gerencial, la creatividad, vivencias, manejo de información sobre a la gestión académica, administrativa, financiera y comunitaria, liderazgo efectivo, comunicación fluida y horizontal, pragmática, holístico y además que permitan concatenarse entre sí para el logro de los objetivos planteados.

Al respecto, Muñoz (2014b), considera que el gerente institucional o directivo: 
Debe ser el líder nato donde exista una toma de decisiones apropiadas a las necesidades e intereses institucionales, donde la motivación este cónsona con el dinamismo social por ello hay renovar las acciones profesionales de los docentes y demás personas que conforman la organización (pág. 176).

Dentro del contexto educativo, lo planificado es la guía a seguir donde se orienta las acciones a seguir en diferentes dimensiones con el apoyo de todos los autores y actores del quehacer educativo involucrados, a fin de lograr los objetivos propuestos, por ello, el directivo debe ser el cuentadante de la puesta en marcha de dicho plan.

Según Hinostroza (2013a):

El directivo de una institución educativa es la base del proceso de enseñanza, porque debe orientarlos, creando una cultura de trabajo colaborativa entre el personal donde estén identificados con la misión y visión institucional, es decir, cada recurso humano debe cumplir un rol determinado de acuerdo a sus habilidades (pág. 54).

En consecuencia, decodificar induce a establecer las jerarquías de importancia en cuanto al manejo de del recurso para cada actividad, de tal manera que se alcancen las metas organizacionales. El directivo debe inducir la institución al diseño organizacional donde se tenga presente lo que se desea lograr, con qué va a contar en cuanto a recursos humanos y materiales y todos y cada una de las tareas que debe ejecutar para dar cumplimiento a lo que solicite la instancia educativa.

\subsubsection{Dimensión Dirección}

El quehacer educativo se desarrolla a partir de la planeación, dirección, ejecución, control y seguimiento de acciones, utilizando las técnicas, instrumentos y medios apropiados para el logro de los objetivos propuestos.

Para Hinostroza (2013b), plantea: "Ios gerentes institucionales deben 
orientar, sensibilizar al personal para lograr los objetivos institucionales a fin de que todos se direccionen a través de un ambiente apropiado de comunicación fluida, respeto y esfuerzo compartido a un fin en particular" (pág. 476).

El directivo como gerente institucional debe presentar un perfil guiado hacia: Enfatizar un liderazgo, haciendo que todos trabajen en función de los objetivos propuestos, orientando y asesorando en todo momento cada una de las acciones, a través de la creatividad, el respeto, la lealtad, entre otros valores que deben ponerse en práctica. Otro valor agregado a este perfil, es determinar el espacio tiempo en que se ejecutará cada una de las acciones a realizar y comprometido en hacer siempre las cosas bien, exaltando que es el ejemplo a seguir dentro de la organización, a su vez, debe ser comunicativo, sensible en la toma de decisiones.

\subsubsection{Gestión pedagógica}

El accionar educativo implica competencias donde se amalgamen conceptos, vivencias, experiencias, aptitudes, conocimientos sobre el quehacer educativo, de esta manera este directivo debe: 1). Manejar el currículo de la institución educativa de acuerdo al nivel que se desarrolla y la emitida por el Ministerio de Educación; 2). Estar en constante evaluación de los materiales concretos que se utilizan en el desarrollo del quehacer educativo a fin de determinar cuáles son los más apropiados para el quehacer educativo.

\subsubsection{En la gestión administrativa}

El directivo debe: a). Manejar la estructura organizativa del plantel, está encargado de la dirección, consejo educativo y otros órganos. Manejar los miembros del personal con sus múltiples diferencias; b). Conocer las normas y estamentos que tipifican el equipamiento institucional; y $\mathrm{c}$ ). Administrar los recursos financieros con un enfoque gerencial. 


\subsubsection{Dimensión control}

Implica que el gerente del centro educativo manejará una serie de elementos básicos: Los estándares de desempeño deben establecerse de acuerdo a las necesidades, midiendo los resultados para finalmente comparar los resultados emitidos, para tomar decisiones pertinentes si hay que cambiar, mejorar o plantear nuevas rutas a seguir.

Los directores, como responsables de las instituciones educativas, deben llevar el control y seguimiento de las acciones a seguir con el fin de realizar los cambios pertinentes, tanto en lo pedagógico, administrativo, financiero y comunitario, donde se tomen las decisiones para enfatizar las metas y logros de objetivos.

\subsection{Determinación de la competencia administrativa}

Dentro de la praxis educativa hay factores esenciales como la eficiencia, eficacia y la productividad donde todos los actores y autores deben estar encauzados en la búsqueda de los objetivos planteados. Tal como lo plantea Alvarado (2015), enfatiza que: "la eficacia implica el logro de los objetivos establecidos donde el personal se sienta satisfecho de lo realizado" (pág. 33). Donde sea resaltante el utilizar la menor cantidad de recursos para cumplir una meta, lo que se denomina eficiencia, es decir, todo el personal de la organización debe cumplir las metas utilizando los recursos racionalmente.

Por otro lado, al lograr la eficiencia y la eficacia determinan la efectividad, implica alcanzar con un bajo costo grandes resultados por parte de quienes hacen vida en la organización. Además, la productividad a nivel educativo implica concatenar las acciones, métodos, herramientas, recursos para lograr los cambios que se estiman en los planes, programas y proyectos de esta manera mejora el rendimiento integral de la institución. Dentro del contexto educativo, se aplica estos cuatro elementos: eficacia, eficiencia, efectividad y productividad, los cuales se plantean como indicadores de 
gestión.

\subsection{Servicio Educativo de Calidad}

A lo largo de la historia la calidad educativa ha permutado de significado esto dependerá de los factores que lo afecten. Al respecto, Valdés (2016), comenta lo siguiente:

A nivel de Latinoamérica la calidad educativa tiene tendencias perceptibles a ser discutidas: La primera procura hacer la discusión, a nivel teórico, la segunda maneja la parte operativa exaltando una serie de indicadores de gestión que conllevan a la calidad y finalmente la tercera no quiere manejarlo, lo evita (págs. 71-72).

\subsection{La calidad integral}

La calidad integral, corresponde a un modelo de calidad promovido por la Organización de las Naciones Unidas para la Educación, la Ciencia y la Cultura (UNESCO), en el que se establece una serie de metas que permiten el desarrollo del Milenio, en el modelo, los países miembros buscan promover una educación de calidad integral que cumpla con los cinco principios establecidos por la UNESCO: relevancia, pertinencia, equidad, eficacia y eficiencia.

\subsubsection{Procesos de acreditación de la calidad}

Según la Ley del Sistema Nacional de Evaluación Acreditación y Certificación de la Calidad Educativa (SINEACE, 2007): está a cargo del IPEBA como ente rector del manejo de estos indicadores de calidad educativa en el nivel de Básica (art. 21); el Instituto Peruano de Evaluación, Acreditación y Certificación de la Calidad de la Educación Básica (IPEBA); gestiona el quehacer educativo, en cuanto a la toma de decisiones en sus diversos procesos a fin de mejorar la praxis educativa y por ende lograr aprendizajes significativos en el educando que induce al desarrollo holístico del individuo. 
En el texto publicado por el Instituto Peruano de Evaluación, Acreditación y Certificación de la Calidad de la Educación Básica (IPEBA), en el año 2011, se considera entre otros aspectos, que para acreditar una institución, implica que los procesos deben estar en constante revisión a fin de mejorarlos, eliminarlos o hacerle las modificaciones pertinentes.

\subsubsection{Autoevaluación}

La autoevaluación, es un proceso que insta a revisar detenidamente los constantes cambios que deben darse en el entorno educativo para que tengan la coherencia y la eficacia y por ende, la formación integral del educando. Este proceso debe realizarse de forma paulatina, y permite a las instituciones educativas una reflexión sobre aquellos elementos a mejorar en el proceso de enseñanza y aprendizaje para generar una retroalimentación que incremente la adquisición de conocimientos.

\subsubsection{Evaluación externa}

Es el segundo nivel que plantea la IPEBA, y debe ser realizada por un ente evaluador autorizado, se aplica el modelo de gestión de calidad, donde se dan las recomendaciones pertinentes a fin de retroalimentar las fortalezas, a fin de mejorar el proceso educativo. Asimismo, se determinan las necesidades e intereses, delimitando avances respecto a los indicadores establecidos.

\subsubsection{Acreditación}

Induce a la acreditación en sí, después de revisar el informe se determina que la institución cuenta con elementos indispensables que conducen a encuentros educativos de calidad. Esta acreditación vence al cabo de cinco años, debido al dinamismo en que se está interactuando el mundo actualmente, por ello debe renovarse, tal como se observa en la figura 1, razón 
por la cual se reiniciará los procesos nuevamente con la visión prospectiva de mejorar la praxis educativa.

Figura 1. Los procesos de la evaluación para la acreditación - IPEBA.

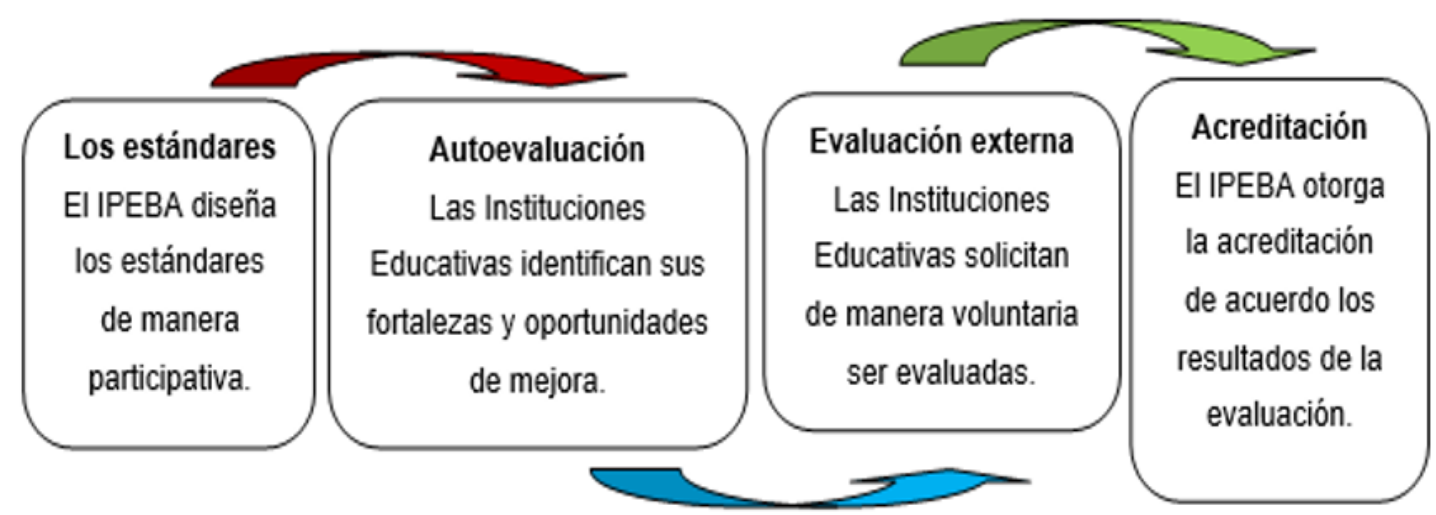

Fuente: La Autora (2019).

A nivel de Perú, las etapas tendrán una duración dependiendo de la realidad política que converja en ese momento histórico social en la cual está inmersa dichas instituciones educativas.

\subsubsection{Los instrumentos para evaluar la calidad}

En realidad, los instrumentos de medición de calidad educativa están estigmatizados a una serie de indicadores que responden a lo planteado por IPEBA. La gestión educativa tiene implícito una serie de variables o llamada también factor que tanto explicito como implícitamente inciden en la mejora de la praxis educativa, lo cual ha sido la respuesta de lo percibido en las diferentes instituciones, el cual perfila la búsqueda de la calidad educativa.

En consecuencia, la calidad educativa tiene relacionado entre sí, porque los estándares permiten elaborar las variables y los indicadores se ejecutan en función del estándar. EI IPEBA, para medir la gestión educativa, planteo: 5 factores, 12 estándares y 43 indicadores; tal como se puede ilustrar en la tabla 1. 
Tabla 1. Factores, estándares e indicadores para evaluar la calidad - IPEBA.

\begin{tabular}{|l|c|c|}
\hline \multicolumn{1}{|c|}{ Factores } & Estándares & Indicadores \\
\hline $\begin{array}{l}\text { Factor 1. } \\
\text { Dirección institucional. }\end{array}$ & 3 & 9 \\
\hline $\begin{array}{l}\text { Factor 2. } \\
\text { Desempeño docente. }\end{array}$ & 3 & 15 \\
\hline $\begin{array}{l}\text { Factor 3. } \\
\text { Trabajo mancomunado familia y comunidad. }\end{array}$ & 2 & 5 \\
\hline $\begin{array}{l}\text { Factor 4. } \\
\text { Uso de la información. }\end{array}$ & 2 & 8 \\
\hline $\begin{array}{l}\text { Factor 5. } \\
\text { Infraestructura y recursos para el aprendizaje. }\end{array}$ & 12 & $\mathbf{4 3}$ \\
\hline TOTAL & 2 &
\end{tabular}

Fuente: La Autora (2019).

La investigación desarrollada correspondió a un diseño no experimental transversal. Estuvo constituida por 80 profesoras que enseñaban en las instituciones educativas del nivel y que pertenecían a la jurisdicción de la UGEL 07. En relación a la muestra, Hernández, Fernández y Baptista (2014), sostienen que: “...la muestra es una parte de la población” (pág. 175); partiendo de un muestreo no probabilístico, porque su elección va a depender de causas relacionadas con el fenómeno.

\section{Resultados}

Luego de la aplicación de los instrumentos a las profesoras, se puede obtener su percepción y el $70 \%$ de la muestra indican que la mayoría de las profesoras de educación inicial han desarrollado competencias administrativas y el $85 \%$ de las profesoras indican que la gestión es de buena calidad, sin embargo, el $5 \%$ de las participantes indicaban que las competencias administrativas de las directoras no eran suficientes para los cargos que ellas ocupaban.

Del análisis de los resultados se puede decir que la gestión 
administrativa en la institución es de calidad, pero requiere mejoras en el personal directivo, por lo tanto, el servicio en la institución es aceptable proponiendo planes de mejoramiento que sería importante desarrollar en un estudio futuro que permita llevar la gestión de buena a excelente donde todos los docentes perciban al personal directivo como buenos administradores.

\section{Conclusiones}

De acuerdo a lo hallado se enfatiza al personal directivo como responsable y principal encargado de la gestión administrativa en los centros escolares de los chorrillos, cuya labor debe ser mejorada mediante la aplicación de mecanismos que optimicen su desempeño dentro del quehacer educativo. Como gerentes, los directivos están en la obligación de diseñar y aplicar políticas que faciliten el proceso educativo donde todos los integrantes de la institución se sientan cómodos y atendidos. Es necesario que se atienda el trato hacia los docentes ya que, en los resultados del instrumento aplicado, si bien existe un balance positivo hay un porcentaje de los encuestados que se siente desatendido y esto puede reflejarse en sus labores diarias haciéndolos sentir desmotivados y por ende afectar de manera directa a los estudiantes. Finalmente, se plantea el diseño de estrategias para lograr el trato equitativo en todos los aspectos del proceso educativo.

\section{Referencias}

Ahumada, J. (1990). En vez de la miseria. 10ma edición, Edición revisada. Santiago de Chile: Ediciones BAT.

Alvarado, M. (2015). El equipo Directivo. Recursos técnicos de gestión. Madrid, España: Editorial Popular.

Beorlegui, C. (2006). La Capacidad Lingüística del Ser Humano: Una Diferencia Cualitativa. THÉMATA. Revista de Filosofía, (37), 139-168, e-ISSN: 2253-900X. Recuperado de: 
http://institucional.us.es/revistas/themata/37/11Beorlegui.pdf

Bonilla, H. (2016). Concepciones del hombre y de la educación en el pensamiento del siglo XX. Madrid, España: Editorial Dykinson, S.L.

Chiavenato, I. (2010). Introducción a la teoría general de la administración. México: McGraw-Hill Interamericana.

Hernández, S., Fernández, C., \& Baptista, P. (2014). Metodología de la Investigación. México: Editorial Cuarta Edición.

Hinostroza, L. (2013a,b). Informe de Progreso Educativo. Lima, Perú: Publicaciones Grade.

Ibáñez, J. (2015a,b). La filosofía de la educación y el futuro de Europa. Madrid, España: Editorial Dykinson, S.L.

Jerez, F. (2016). Educación, filosofía de la educación y contexto. Madrid, España: Editorial Dykinson, S.L.

López, C. (2019). Practica Educativa en la Educación Primaria desde la Perspectiva de la Pedagogía del Amor. Revista Scientific, 4(11), 280294, e-ISSN: 2542-2987. Recuperado de:

https://doi.org/10.29394/Scientific.issn.2542-2987.2019.4.11.14.280-

$\underline{294}$

Muñoz, E. (2014a,b). Tecnología de la organización educativa. Barcelona, España: Editorial OI Kostau, S.A.

Reyes, S. (2015). Estrategias Metodológicas. El Aula. Lima, Perú: Editorial San Marcos.

SINEACE (2007). Artículo 21. Ley del Sistema Nacional de Evaluación, Acreditación y Certificación de la Calidad Educativa № 28740. Lima, Perú: El Congreso de la República.

Sovero, S. (2014). Pasos para elaborar proyectos y tesis de Investigación Científica. Lima, Perú: Editorial San Marcos.

Valdés, H. (2016). Reflexiones en torno a la evaluación de la calidad educativa en América Latina y el Caribe. Santiago, Chile: 
Organización de las Naciones Unidas para la Educación, la Ciencia y la Cultura - UNESCO; Laboratorio Latinoamericano de Evaluación de la Calidad de la Educación - LLECE.

Werther, C. (2016). Técnicas para investigar: recursos metodológicos para la preparación de proyectos de investigación. $3^{a}$ edición. Córdoba, Argentina: Editorial Brujas. 
Rocío Soledad Riffo San Martín

e-mail: rocio.riffo@profe.umcervantes.cl

Nacida en Chile, el 17 de enero del año 1983.

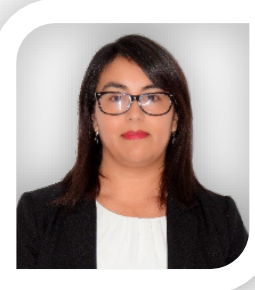

Doctorando en educación; Magister en Educación;

Profesora de Educación General Básica; Licenciada

en Educación; Académica de la Universidad Miguel de

Cervantes (UMC); Posteriormente he realizado

funciones en instituciones del ámbito educativo y

académico como Directora de proyectos educativos; y actualmente como Coordinadora y docente en el programa de Magister en Educación de la Universidad Miguel de Cervantes (UMC).

El contenido de este manuscrito se difunde bajo una Licencia de Creative Commons ReconocimientoNoComercial-Compartirlgual 4.0 Internacional 